\title{
Heavy ion abundances and spectra from the large solar energetic particle events of October-November 2003
}

\author{
C. M. S. Cohen, ${ }^{1}$ E. C. Stone, ${ }^{1}$ R. A. Mewaldt, ${ }^{1}$ R. A. Leske, ${ }^{1}$ A. C. Cummings, ${ }^{1}$ \\ G. M. Mason, ${ }^{2,3}$ M. I. Desai, ${ }^{2}$ T. T. von Rosenvinge, ${ }^{4}$ and M. E. Wiedenbeck ${ }^{5}$ \\ Received 4 January 2005; revised 27 April 2005; accepted 9 May 2005; published 10 August 2005.
}

[1] Observations from the Solar Isotope Spectrometer and the Ultra Low Energy Isotope Spectrometer on the ACE spacecraft during the extremely large events of October and November 2003 are combined to create heavy ion spectra over more than 3 decades in energy. The resulting spectra differed substantially in shape from event to event, as well as from element to element within a given event, resulting in energy-dependent abundance ratios. Although the effects of strong local shock acceleration are apparent in the intensities of the 28 and 29 October events, these do not explain the order of magnitude differences between the event-integrated abundances obtained at $0.64-0.91 \mathrm{MeV} /$ nucleon and those at $12-60 \mathrm{MeV} /$ nucleon. The higher-energy abundances relative to the lower-energy ones show trends with nuclear charge or charge-to-mass ratio that are similar for all the events and suggest that heavier ions are less efficiently accelerated to high energies. The position of the breaks in the energy spectra of $\mathrm{O}, \mathrm{Ne}, \mathrm{Mg}, \mathrm{Si}, \mathrm{S}, \mathrm{Ca}$, and $\mathrm{Fe}$ can be understood in terms of leakage from the shock region, if the mean free path is assumed to be a power law in rigidity. The resulting rigidity dependence is consistent with a source of wave turbulence in the vicinity of the shock when the ions are accelerated.

Citation: Cohen, C. M. S., E. C. Stone, R. A. Mewaldt, R. A. Leske, A. C. Cummings, G. M. Mason, M. I. Desai, T. T. von Rosenvinge, and M. E. Wiedenbeck (2005), Heavy ion abundances and spectra from the large solar energetic particle events of October-November 2003, J. Geophys. Res., 110, A09S16, doi:10.1029/2005JA011004.

\section{Introduction}

[2] To call the period of 25 October to 11 November 2003 a solar "active" period would be an extreme understatement. During these 18 days, there were 43 coronal mass ejections (CMEs) identified with the LASCO coronagraph on SOHO, 22 of which had speeds in excess of $800 \mathrm{~km} / \mathrm{s}$ [Gopalswamy et al., 2005]. There were also 8 X-class flares, including the largest ever recorded on 4 November (http:// www.sec.noaa.gov/Data/index.html). Not surprisingly, these flares and CMEs gave rise to a series of large solar energetic particle (SEP) events, some of the largest of this solar cycle. At $\sim 50 \mathrm{MeV} /$ nucleon, oxygen intensities show 5 distinct events (Figure 1), 4 of which can be matched to fast CMEs associated with flares occurring in active region 10486 as it moved across the solar disk (the first event matches a flare in active region 10484; see Table 1). Just upstream of the Earth at least 7 interplanetary shocks were observed with shock normal angles ranging from $14^{\circ}$ to $80^{\circ}$ (Table 2).

\footnotetext{
${ }^{1}$ Space Radiation Laboratory, California Institute of Technology, Pasadena, California, USA.

${ }^{2}$ Department of Physics, University of Maryland, College Park, Maryland, USA.

${ }^{3}$ Also at Institute for Physical Science and Technology, University of Maryland, College Park, Maryland, USA.

${ }^{4}$ NASA Goddard Space Flight Center, Greenbelt, Maryland, USA.

${ }^{5}$ Jet Propulsion Laboratory, Pasadena, California, USA.

Several of these shocks were still accelerating particles at $1 \mathrm{AU}$ as evidenced by the coincident increases in lowenergy oxygen intensities (Figure 1).

[3] The October/November 2003 time period provides an unusual opportunity to examine a series of SEP events originating from the same active region as it moved across the solar disk. During this transit several aspects influencing SEP acceleration, such as magnetic connection to the flare longitude, available seed population, and shock strength/ orientation/etc., changed significantly. Whether the impact of such variations can be seen in the energetic particle composition and spectra, or possibly inferred from the observations, is worth investigating as it furthers the understanding of the generation of large SEP events and their characteristics.

\section{Instrumentation and Data Analysis}

[4] The Advanced Composition Explorer (ACE) resides near the L1 Lagrangian point between the Earth and the Sun, providing a platform to study energetic particles and plasma conditions that will shortly impact the Earth [Stone et al., 1998b]. The energy spectra and elemental, isotopic, and ionic charge composition of energetic particles from many large SEP events have been measured by the ACE sensors since September 1997. The heavy ion data presented here are from two of the energetic particle detectors on the spacecraft: the Ultra-Low Energy Isotope Spectrometer (ULEIS) [Mason et al., 1998] and the Solar Isotope 


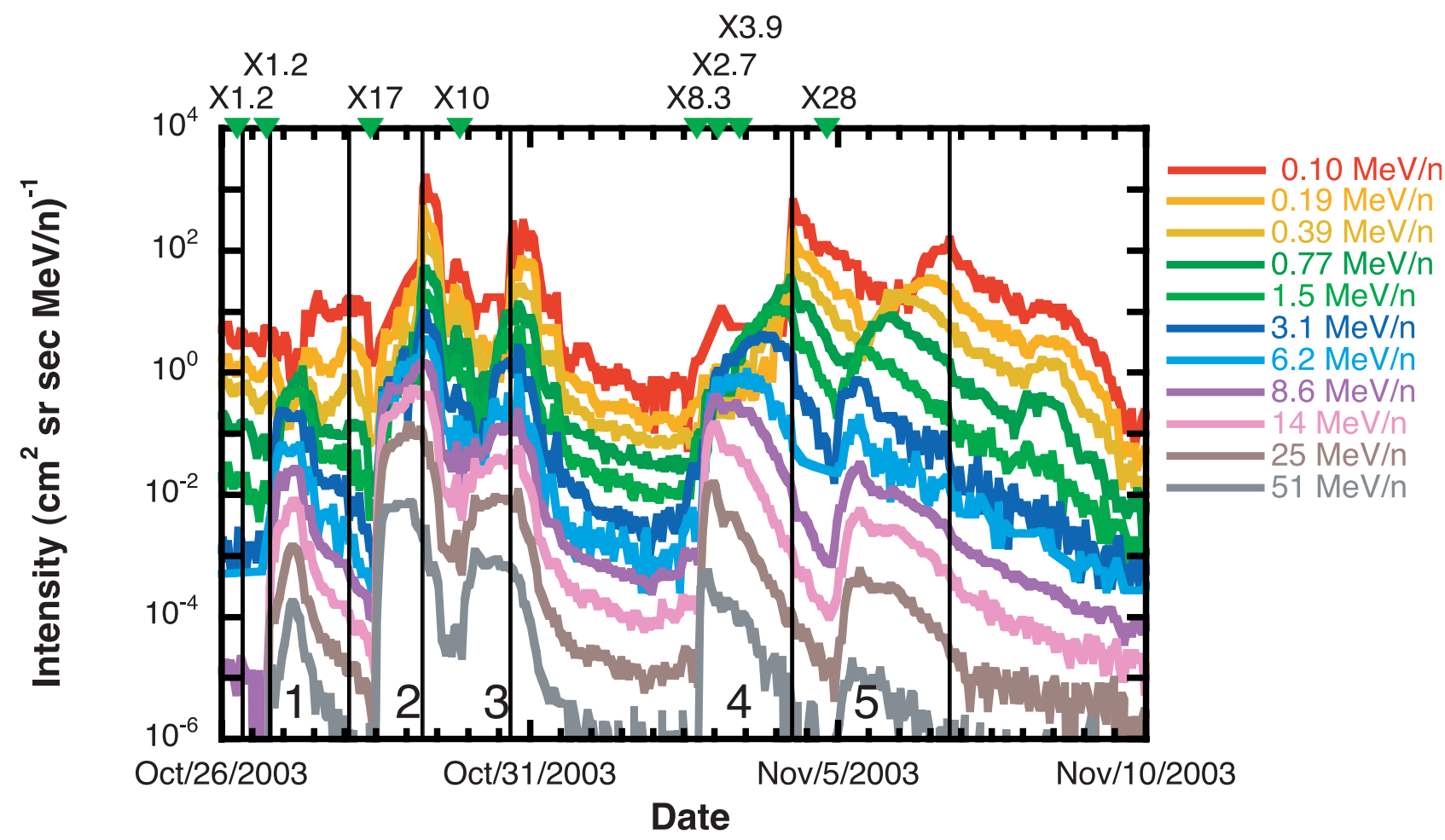

Figure 1. Hourly oxygen intensity plotted versus time for several of the ULEIS and SIS energy bands. Flare start times are indicated by the inverted triangles at the top of the plot (with X-ray flare size given above). Shock passages are denoted by vertical solid lines. The five identified events are numbered for reference.

Spectrometer (SIS) [Stone et al., 1998a]. ULEIS utilizes time-of-flight and energy measurements to determine ion mass for ions from $\sim 0.1$ to $8 \mathrm{MeV} /$ nucleon, while SIS employs $d E / d x$ versus residual energy measurements to obtain nuclear charge, mass, and total energy for ions from $\sim 6$ to $160 \mathrm{MeV} /$ nucleon. The excellent resolution and large geometry factors of these two instruments allow spectra for ions from $\mathrm{C}$ to $\mathrm{Fe}$ to be accurately determined over a wide energy range.

[5] Five SEP events are readily identified in the $\sim 50 \mathrm{MeV} /$ nucleon oxygen intensities shown in Figure 1. In the SIS energy range there is negligible velocity dispersion in the onsets of the different energy channels, so fluences are calculated over simple time intervals as given in Table 3. As can be seen from Figure 1, the time profiles of lower-energy ions measured by ULEIS are significantly different from those at SIS energies, a characteristic that is discussed more in section 5. Because of the substantial energy dispersion seen at ULEIS energies, the time intervals for each event are energy-dependent and determined from examining particle velocity versus time spectrograms [e.g., see Mason et al., 2000, Figure 1]. For reference Table 3 includes the time intervals used for the ULEIS data at 0.1 and $0.77 \mathrm{MeV} /$ nucleon. Since the fluences obtained from the ULEIS data are dominated by the peak intensity time periods, the results are not very sensitive to the details of the energy-dependent time intervals.

[6] During time periods of high particle intensity, an automatic door reduces the opening aperture of ULEIS to help prevent saturation and dead time problems in the instrument. The door was $100 \%$ open for event $1,1 \%$ open during events 2,3 , and 4 , and $6 \%$ open during event 5 . Even with a $1 \%$ door position, there were significant dead time effects from $\sim 1020 \mathrm{UT}, 28$ October, through $1300 \mathrm{UT}, 29$

Table 1. Related Solar Event Characteristics

\begin{tabular}{|c|c|c|c|c|c|c|c|c|}
\hline \multirow[b]{2}{*}{ Event } & \multicolumn{5}{|c|}{ Flare $^{\mathrm{a}}$} & \multicolumn{3}{|c|}{$\mathrm{CME}^{\mathrm{b}}$} \\
\hline & Date & Start Time, UT & Size & Longitude & Region & Time, UT & Speed & Width \\
\hline 1 & 26 Oct & 1721 & $\mathrm{X} 1.2$ & W38 & 10484 & 1754 & 1537 & $171^{\circ}$ \\
\hline 2 & 28 Oct & 0951 & X17 & E08 & 10486 & 1110 & 2459 & Halo \\
\hline 3 & 29 Oct & 2037 & X10 & W02 & 10486 & 2054 & 2029 & Halo \\
\hline 4 & $2 \mathrm{Nov}$ & 1703 & X8.3 & W56 & 10486 & 1730 & 2598 & Halo \\
\hline 5 & $4 \mathrm{Nov}$ & 1929 & X28 & W83 & 10486 & 1954 & 2657 & Halo \\
\hline
\end{tabular}

${ }^{a}$ Data are from http://www.sec.noaa.gov/Data/index.html.

${ }^{\mathrm{b}}$ Data are from Gopalswamy et al. [2005]. 
Table 2. Shock Parameters ${ }^{\mathrm{a}}$

\begin{tabular}{ccccccc}
\hline $\begin{array}{c}\text { Associated } \\
\text { Event }\end{array}$ & Date & $\begin{array}{c}\text { Shock Time, } \\
\text { UT }\end{array}$ & $\theta_{B n}, \mathrm{deg}$ & Velocity, km/s & $\begin{array}{c}\text { Mach } \\
\text { Number }\end{array}$ & $\begin{array}{c}\text { Transit Time, }^{\text {b }} \\
\text { hours }\end{array}$ \\
\hline & 26 Oct & 0809 & & & & \\
& 26 Oct & 1832 & $80 \pm 3$ & $631 \pm 150$ & $1.3 \pm 0.1$ & 32 \\
1 & 28 Oct & 0131 & 68 & & & 19 \\
2 & 29 Oct & 0558 & 14 & & & 19 \\
3 & 30 Oct & 1619 & 54 & & & 36 \\
4 & 4 Nov & 0559 & $43 \pm 4$ & $779 \pm 34$ & $4.4 \pm 0.7$ & 47 \\
5 & 6 Nov & 1919 & $66 \pm 6$ & $524 \pm 40$ & $3.0 \pm 0.7$ & \\
\hline
\end{tabular}

${ }^{\mathrm{a}}$ Data are from http://www-ssg.sr.unh.edu/mag/ace/ACElists/obs_list.html.

${ }^{\mathrm{b}}$ Time is measured relative to first observed time of the corresponding CME.

October, due to penetration of the instrument walls by highenergy particles. Dead time corrections for this period were calculated by comparing the ULEIS and SIS oxygen intensities at overlapping energy intervals.

\section{Five Large Events}

[7] The largest of the 5 events studied here, starting on 28 October, is also one of the most intense events seen by the SIS instrument since launch in August, 1997. Figure 2 shows hourly intensities of $10 \mathrm{MeV} /$ nucleon oxygen for a 4 day period centered around the time of peak intensity for 5 of the largest events measured by SIS. Although the peak is brief, the intensity of the 4 November 2001 event is slightly higher than that of 28 October $2003\left(0.80\right.$ versus $0.79\left(\mathrm{~cm}^{2}\right.$ sr s MeV/nucleon $)^{-1}$ ). The fact that the peak intensities of $10 \mathrm{MeV} /$ nucleon oxygen are all within $17 \%$ of each other for the 28 October 2003, 14 July 2000 and 4 November 2001 events may indicate the streaming limit, where the selfgenerated waves reach high enough intensities to inhibit the transport of the particles [Reames and $\mathrm{Ng}, 1998$ ], is being approached in these events. When the event-integrated fluence is calculated as a function of energy (Figure 3), it is clear that the 28 October event is the largest by $\geq 30 \%$ at $10 \mathrm{MeV} /$ nucleon.

[8] In a broader context, the fluence of this event can be compared to measurements made with different instruments in the past (although several of the very largest events, for example, 4 August 1972 and 23 February 1956, were not observed with instruments capable of measuring oxygen fluences). This is done for the events of 6 November 1997 , 2 November 1992, 23 September 1978, and 19 October 1989 in Figure 3. The 28 October 2003 event is second to the 19 October 1989 event over the energies measured; however, if one were to extrapolate to energies above $100 \mathrm{MeV} /$ nucleon it is possible that the 28 October 2003 event would surpass 19 October 1989. Interestingly, significantly smaller events such as 6 November 1997 and
2 November 1992 have substantially harder spectra which could result in them being the events of greatest significance at energies of $\sim 500 \mathrm{MeV} /$ nucleon and greater.

[9] This variability is apparent even for the 5 events of October/November 2003. The fluence spectra for each event are determined from 0.1 to $80 \mathrm{MeV} /$ nucleon by combining the observations of the ULEIS and SIS instruments (Figure 4). Although all the events have a bend in the spectra near 1-10 MeV/nucleon, their behavior at energies on either side of this bend differs significantly. Although the "power law $\times$ exponential" spectral form was suggested by Ellison and Ramaty [1985] to describe the spectral shape at the shock, it has been used with success to describe eventintegrated fluences of SEPs in the past [e.g., Tylka et al., 2001]. However, here it appears to adequately describe the iron and oxygen spectra only of the second event (28 October). An expectation of this form (which we will refer to as "ER-type spectra") is that the $e$-folding energy for different elements is correlated with the ion charge-to-mass $(Q / M)$ ratios. This generally results in strongly falling $\mathrm{Fe} / \mathrm{O}$ ratios with increasing energies [Tylka, 2001], as is seen for this event (Figure 5).

[10] Another common spectral shape is a broken power law [Tylka et al., 2005; Mewaldt et al., 2005], with element-independent spectral indices on either side of a transition region which is usually referred to as the spectral break (even though the "break" is typically not sharp). Although none of the event-integrated spectra fit this form exactly, the 29 October event does have Fe/O ratios which are approximately constant on either side of the spectral break. Events on 26 October and 2 November have $O$ and Fe spectra with differing shapes, producing large energy dependencies in their $\mathrm{Fe} / \mathrm{O}$ ratios (Figure 5). This characteristic is even more extreme in the 4 November event with $\mathrm{Fe} / \mathrm{O}$ ratios varying almost 2 orders of magnitude over the energy range measured.

[11] By integrating the elemental spectra over two energy ranges, the composition for many elements can be com-

Table 3. SIS and ULEIS Time Intervals

\begin{tabular}{|c|c|c|c|c|c|c|c|c|c|c|c|c|}
\hline \multirow[b]{2}{*}{ Event } & \multicolumn{4}{|c|}{$\geq 7 \mathrm{MeV} /$ nucleon } & \multicolumn{4}{|c|}{$0.1 \mathrm{MeV} /$ nucleon } & \multicolumn{4}{|c|}{$0.77 \mathrm{MeV} /$ nucleon } \\
\hline & $\begin{array}{l}\text { Start } \\
\text { Date }\end{array}$ & $\begin{array}{c}\text { Start Time, } \\
\text { UT }\end{array}$ & $\begin{array}{l}\text { Stop } \\
\text { Date }\end{array}$ & $\begin{array}{c}\text { Stop Time, } \\
\text { UT }\end{array}$ & $\begin{array}{l}\text { Start } \\
\text { Date }\end{array}$ & $\begin{array}{c}\text { Start Time, } \\
\text { UT }\end{array}$ & $\begin{array}{l}\text { Stop } \\
\text { Date }\end{array}$ & $\begin{array}{c}\text { Stop Time, } \\
\text { UT }\end{array}$ & $\begin{array}{l}\text { Start } \\
\text { Date }\end{array}$ & $\begin{array}{c}\text { Start Time, } \\
\text { UT }\end{array}$ & $\begin{array}{l}\text { Stop } \\
\text { Date }\end{array}$ & $\begin{array}{c}\text { Stop Time, } \\
\text { UT }\end{array}$ \\
\hline 1 & 26 Oct & 1447 & 28 Oct & 0000 & 27 Oct & 0135 & 27 Oct & 1424 & 26 Oct & 1816 & 27 Oct & 1424 \\
\hline 2 & $28 \mathrm{Oct}$ & 1030 & 29 Oct & 1837 & $28 \mathrm{Oct}$ & 2047 & 29 Oct & 1800 & $28 \mathrm{Oct}$ & 1328 & 29 Oct & 1800 \\
\hline 3 & $29 \mathrm{Oct}$ & 2100 & 31 Oct & 0933 & 30 Oct & 1136 & 31 Oct & 1200 & $30 \mathrm{Oct}$ & 0756 & 31 Oct & 1200 \\
\hline 4 & 2 Nov & 1655 & $4 \mathrm{Nov}$ & 1725 & 3 Nov & 0359 & 4 Nov & 2136 & $2 \mathrm{Nov}$ & 2040 & $4 \mathrm{Nov}$ & 2136 \\
\hline 5 & $4 \mathrm{Nov}$ & 2131 & $7 \mathrm{Nov}$ & 1200 & $5 \mathrm{Nov}$ & 0847 & $7 \mathrm{Nov}$ & 1200 & $5 \mathrm{Nov}$ & 0128 & $7 \mathrm{Nov}$ & 1200 \\
\hline
\end{tabular}




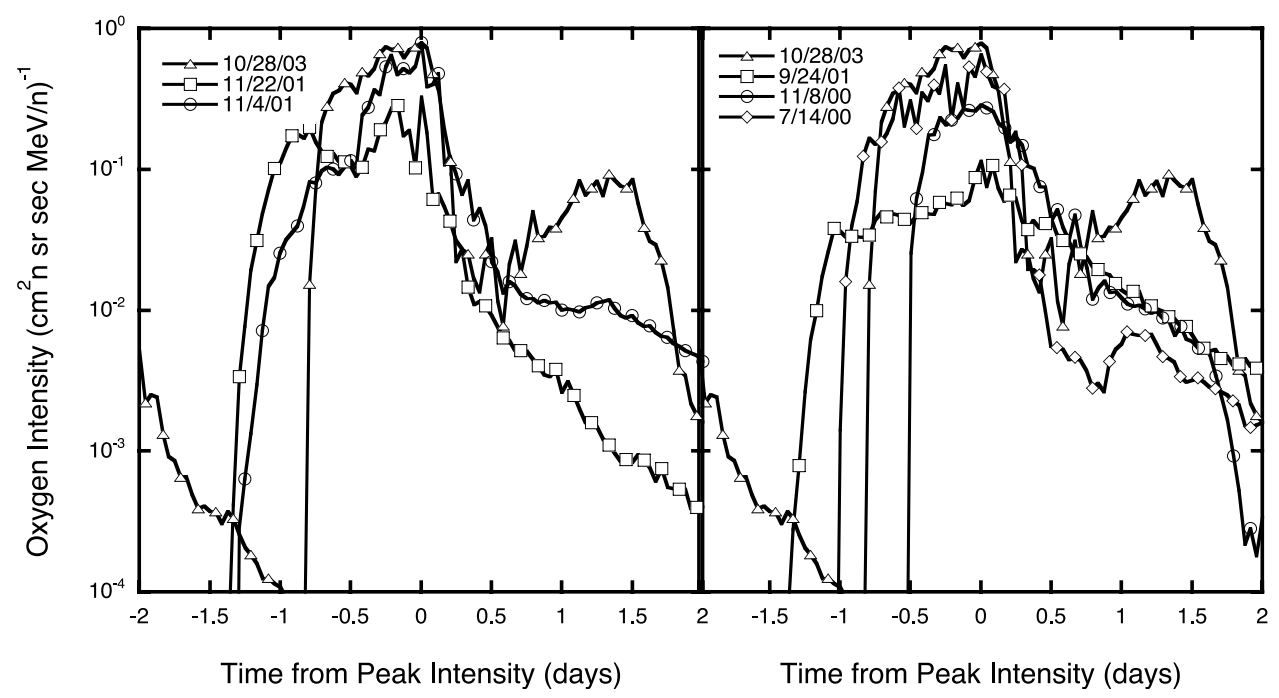

Figure 2. Hourly intensities of $10 \mathrm{MeV} /$ nucleon oxygen plotted versus time (centered at the time of peak intensity) for six large events measured by SIS during solar cycle 23.

pared for the 5 events. In the top plots of Figure 6 , the abundances from ULEIS for 0.64 to $0.91 \mathrm{MeV} /$ nucleon and SIS from 12 to $60 \mathrm{MeV} /$ nucleon, relative to oxygen, are given as a function of nuclear charge. The bottom plots are further normalized to the average large SEP event abundances of Reames [1999], which were determined from measurements of 3-7 MeV/nucleon ions and are often used as the standard for large SEP event composition. Although there are significant differences between events (particularly for Ar-Ni), the dissimilarity between the abundance patterns at the two energies is most striking. All the events are enhanced (relative to the typical values of Reames) in ions heavier than $\mathrm{Ne}$ at ULEIS energies, while three of the five events are depleted in ions heavier than $\mathrm{Si}$ at SIS energies.

[12] While Mazur et al. [1992] showed that abundance variability can increase with increasing energy, the event-toevent variation in the October-November 2003 events is nearly as large near $1 \mathrm{MeV} /$ nucleon as it is above $10 \mathrm{MeV} /$ nucleon. For $\mathrm{Ca}-\mathrm{Ni}$ at $12-60 \mathrm{MeV} /$ nucleon, the events divide into two groups, with events 1 and 3 having close to nominal abundances and events 2, 4, and 5 being depleted in heavy ions. A corresponding pattern does not appear at $0.64-0.91 \mathrm{MeV} /$ nucleon, where the variation is more uniform. Indeed, even the ordering of the events by increasing $\mathrm{X} / \mathrm{O}$ ratio (e.g., $\mathrm{Fe} / \mathrm{O}$ or $\mathrm{C} / \mathrm{O}$ ) is not preserved

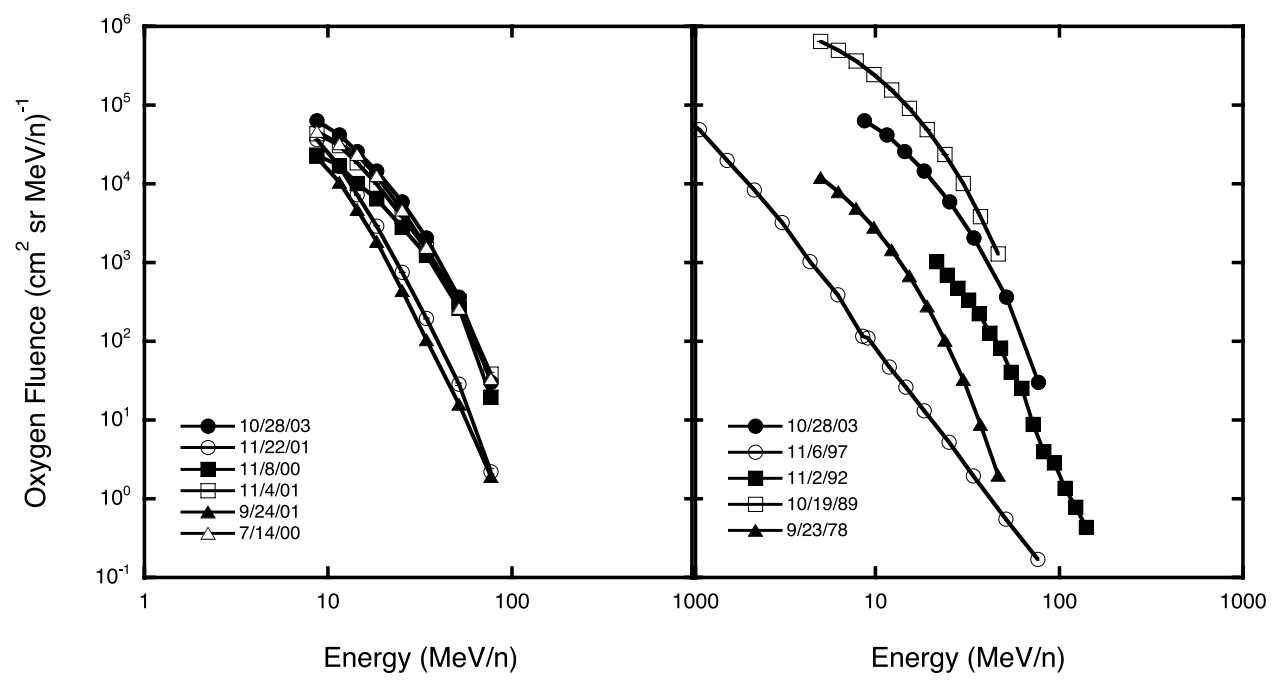

Figure 3. Oxygen fluences plotted versus energy for (left) the same six events given in Figure 2 and (right) other historically large events. The 28 October 2003 event is given in both plots for comparison. Data for the 1978, 1989, and 1992 events are from Cook [1981], Garrard and Stone [1991], and Williams [1998], respectively. All other data are from ACE/SIS (and ACE/ULEIS for the 6 November 1997 event [Mason et al., 1999]). 


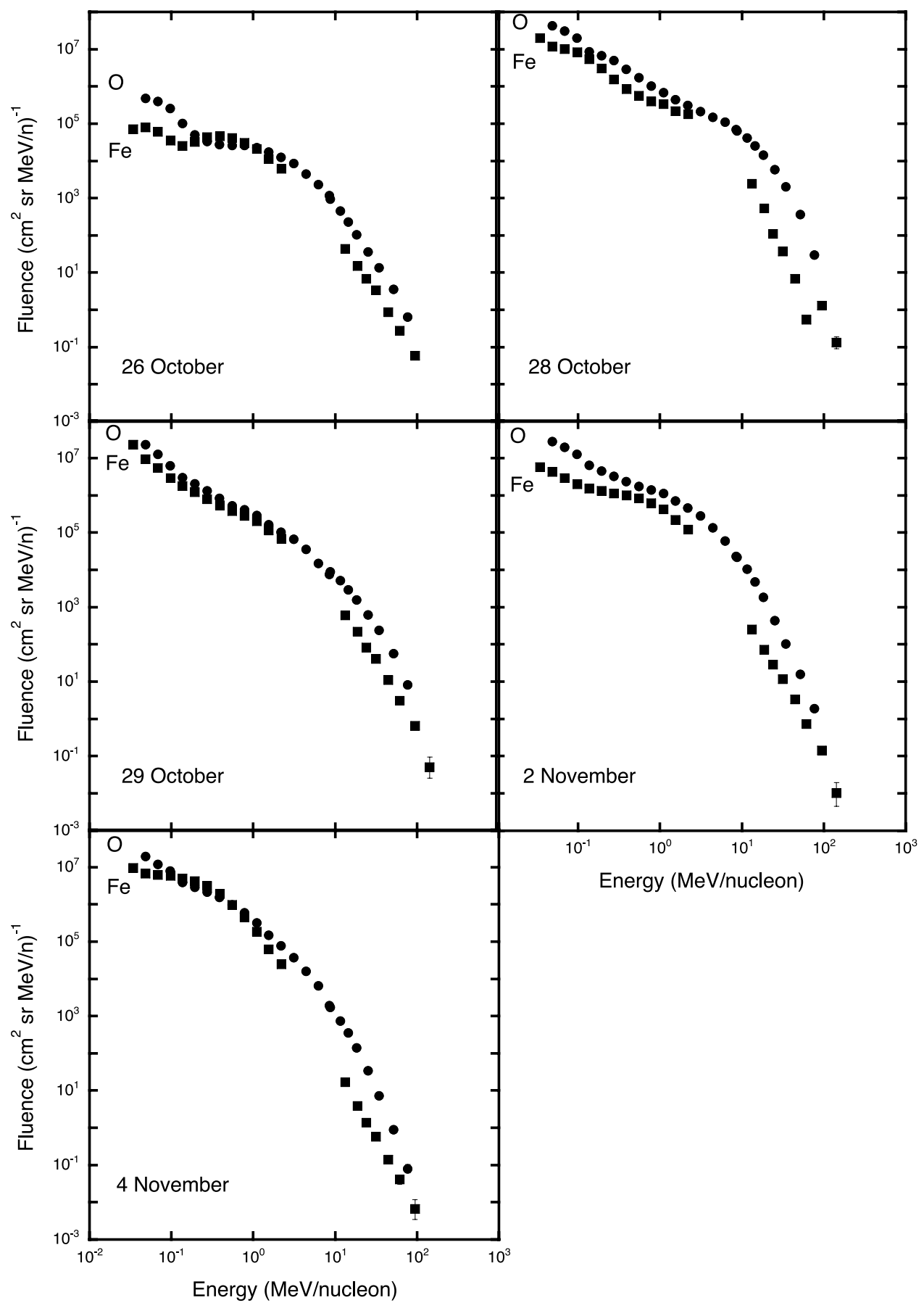

Figure 4. Event-integrated oxygen (circles) and iron (squares) fluences plotted versus energy for each of the five events. The gaps in the Fe spectra (from $\sim 3$ to $10 \mathrm{MeV} /$ nucleon) are due to different measurement techniques of the ULEIS and SIS sensors.

between the two energy intervals; that is, the events that have the highest $\mathrm{Si} / \mathrm{O}$ ratios at lower energies are not the same events that are richest in $\mathrm{Si} / \mathrm{O}$ at higher energies.

\section{Shock Spectra Evolution}

[13] Shock passages were observed during all 5 SEP events. One had concurrent increases in the oxygen particle intensities up to $\sim 0.5 \mathrm{MeV} /$ nucleon (4 November) and two of them had increases up to $\sim 5 \mathrm{MeV} /$ nucleon (29 and 30
October), suggesting significant local particle acceleration. The hourly oxygen spectra for 5 hours before and after the passages of these shocks are shown in Figure 7; in all cases the spectral shapes after the shock passage (open symbols) are fairly invariant and reasonably well characterized by broken power laws or ER-type spectra. The spectral shapes of many elements, in addition to oxygen, for two of these shocks are discussed by Mewaldt et al. [2005], where the authors discuss the organization of the break points of the spectra as a function of nuclear charge and $Q / M$. 


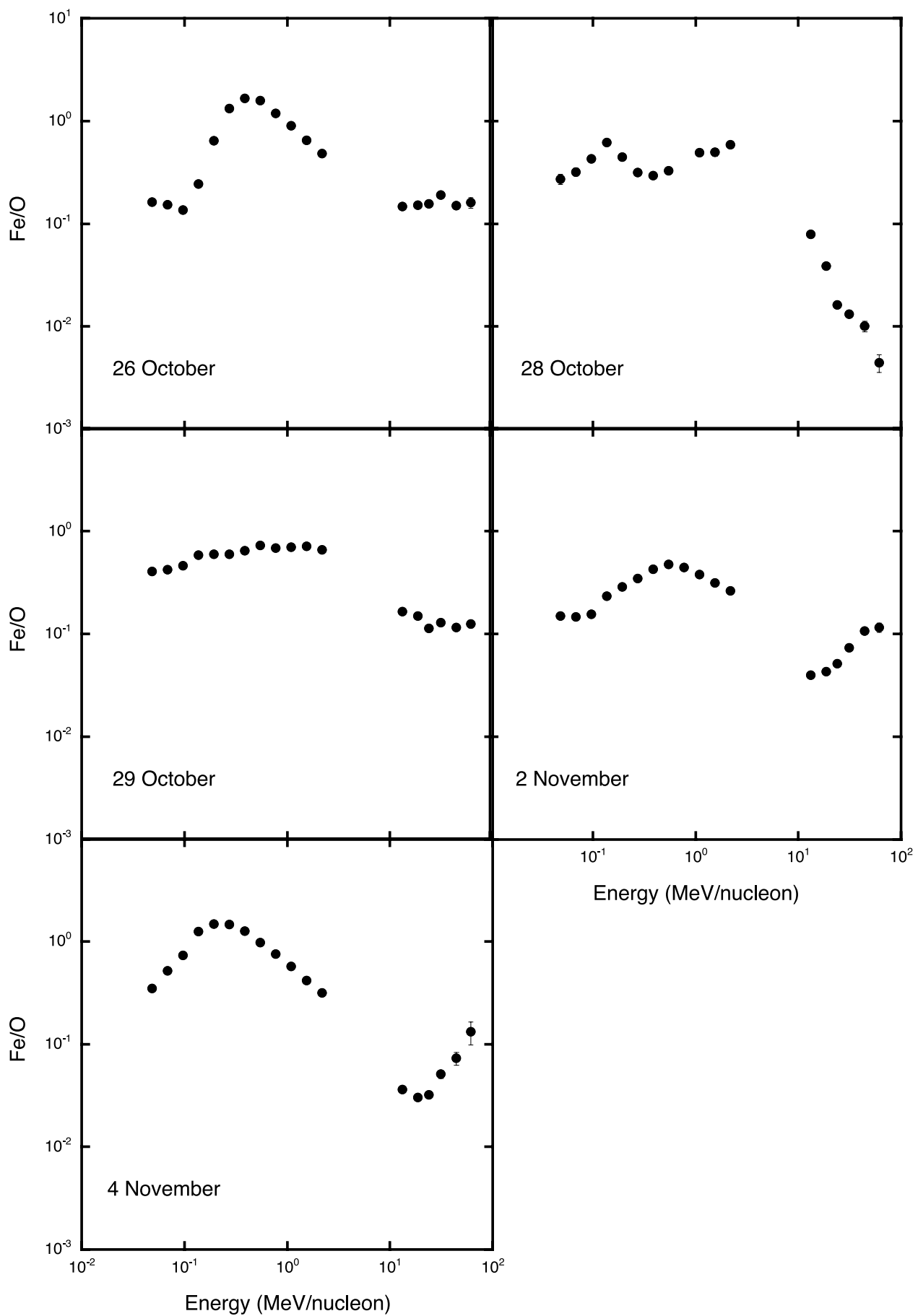

Figure 5. Iron-to-oxygen fluence ratios plotted as a function of energy for the five events.

[14] In the 29 and 30 October and 4 November shock passages, moving from -5 hours to -1 hour, the spectra unroll with the intensities increasing at lower energies until the arrival of the shock. By contrast, the spectra above $\sim 10 \mathrm{MeV} /$ nucleon remain approximately unchanged. A strong difference between the three shock passages is the energy $\left(E_{d}\right)$ at which the -5 hour spectrum diverges from the postshock spectra: nearly $10 \mathrm{MeV} /$ nucleon for the 29 October shock, $\sim 1 \mathrm{MeV} /$ nucleon for the 30 October shock, and below $1 \mathrm{MeV} /$ nucleon for the 4 November shock. In comparison, there is no such unrolling seen in the 28
October and 6 November shock passages. Although the spectral unrolling appears similar to that seen in outer heliospheric observations of anomalous cosmic rays (ACRs) [Cummings and Stone, 1998], the primary cause of the rollovers seen here is not exclusively the rigidity dependence of the ion mean free paths as it is for the ACR spectra. This unrolling is a consequence of the fact that the lower-energy particles take longer to travel the same distance as those at the higher energies (velocity dispersion) combined with the fact that the lower-energy ions have shorter mean free paths and so diffuse shorter distances 


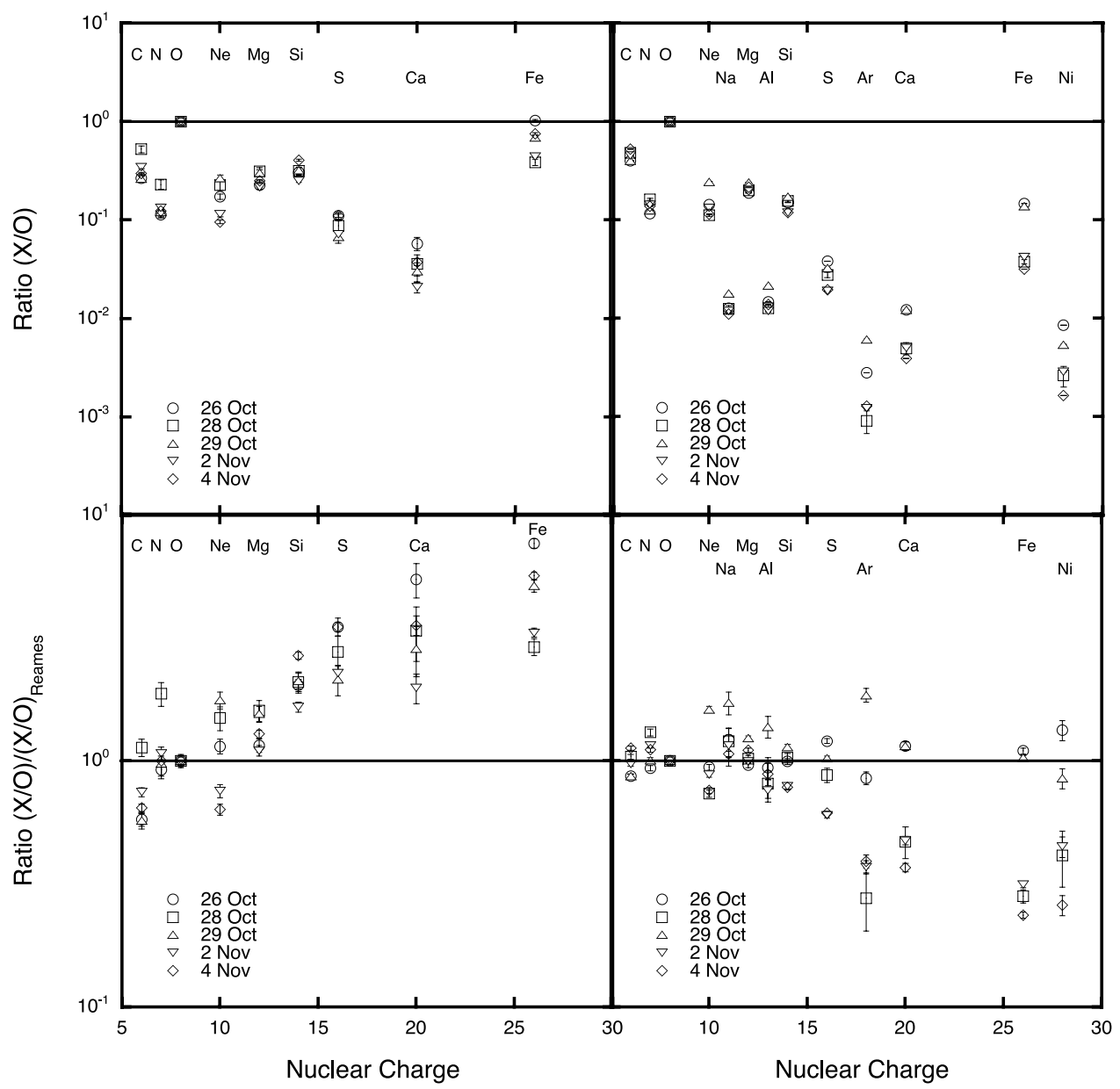

Figure 6. Event-integrated abundances relative to oxygen plotted versus nuclear charge for two different energy ranges: (left) $0.64-0.91 \mathrm{MeV} /$ nucleon from ULEIS and (right) $12-60 \mathrm{MeV} /$ nucleon from SIS. The bottom plots are further normalized to the large SEP event abundances of Reames [1999].

upstream than higher-energy particles (diffusive dispersion). Both effects result in the higher-energy ions being detected earlier than the lower-energy ions and the collective effect can be seen in the timing of the peak intensities during the 4 November event (event 5) in Figure 1.

[15] By the time the shocks of 28 October and 6 November arrive, even the low-energy particle intensities have peaked and so no unrolling of the spectrum is seen near the shock passage. In contrast, the fast shocks of 29, 30 October and 4 November arrive and fill in the low energy portion of the spectrum earlier than would be the case because of dispersion alone. The value of $E_{d}$ is largely a function of the amount of dispersion (related to the distance traveled by the ions, the amount of upstream turbulence, and the magnetic connection to the shock) and how quickly the shock arrives. Since the transit times of the 29 and 30 October shocks were the same (see Table 2), the difference in the $E_{d}$ values (10 MeV/nucleon versus $1 \mathrm{MeV} /$ nucleon) suggests there was more dispersion (i.e., a larger delay in the arrival time of low-energy ions relative to high-energy ions) in event 2 as compared to event 3 , although it is difficult to verify this from Figure 1. Alternatively, the difference in $E_{d}$ may be related to the turbulence around the shock and the strength of the shock itself. The magnetic field increased at the shock passage on 29 October by $30 \mathrm{nT}$ compared to $8 \mathrm{nT}$ for the 30 October shock (http://www-ssg.sr.unh.edu/mag/ace/ ACElists/obs_list.html).

[16] The contribution of locally accelerated ions from these shocks to the event-integrated fluences is discussed in section 5, where an attempt is made to understand the differences among the 5 SEP events. The effects of escape from the shock and interplanetary transport are also considered. The key observations to be explained are (1) the substantially varied spectral shapes from event to event, (2) the different $\mathrm{O}$ and $\mathrm{Fe}$ spectral shapes within each event, and (3) the resulting energy-dependent composition (which also varies in trend from one event to the next).

\section{Discussion}

[17] Although 4 of the 5 events studied here were related to flares that originated in the same active region, the oxygen and iron spectra differ significantly from one event to the next. That the shapes of the oxygen and iron spectra are different within each event gives rise to energy-dependent $\mathrm{Fe} / \mathrm{O}$ ratios. It can also be inferred from the changing abundance patterns with respect to nuclear charge $(Z)$ seen at two energy intervals that the spectral shapes of other 


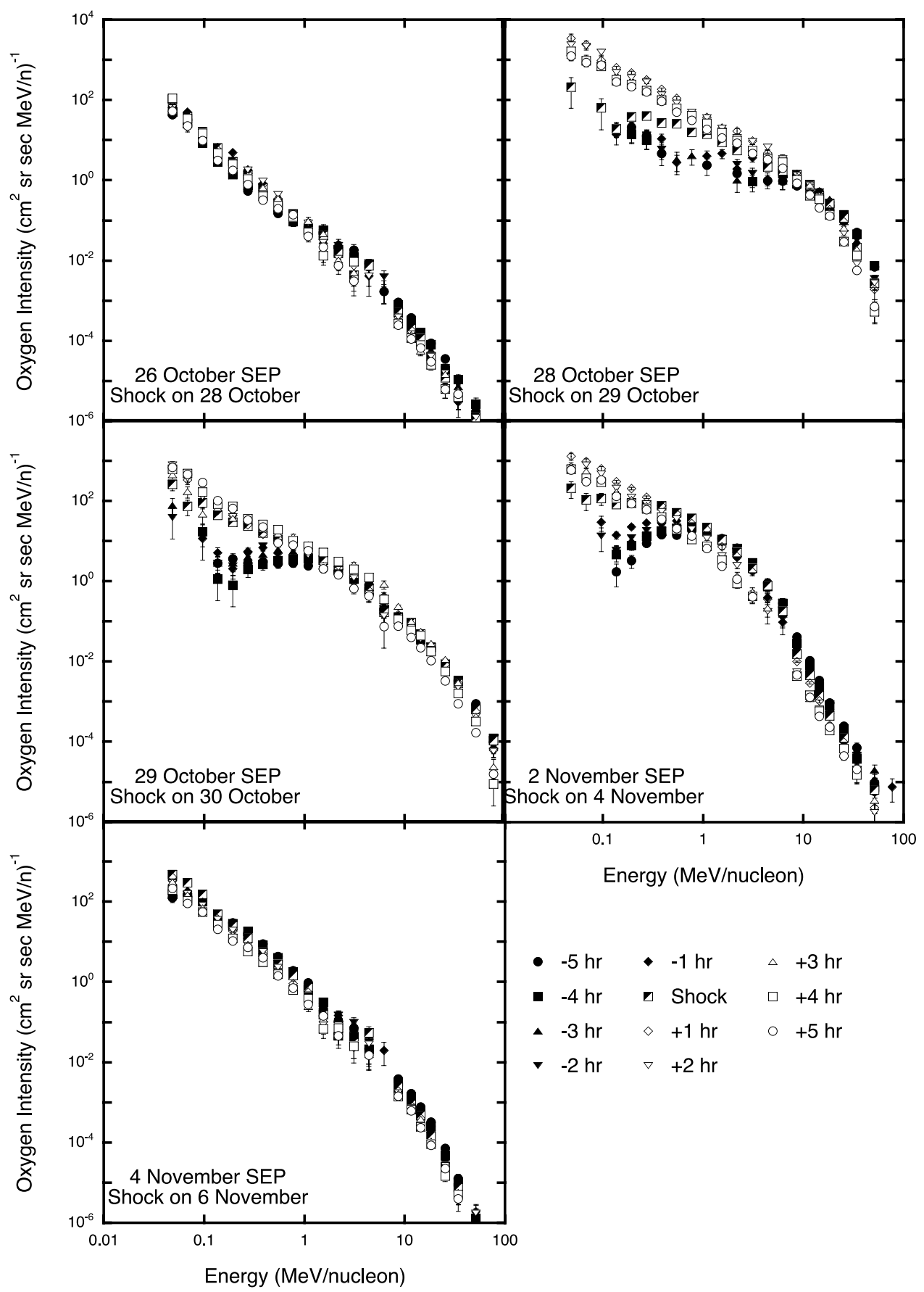

Figure 7. Hourly oxygen spectra plotted versus energy for \pm 5 hours around the passage shocks during the five SEP events (dates of the shock passages are given on the plots). Filled symbols indicate spectra before the shock passage, open symbols denote spectra after the shock passage, and the half-filled squares indicate spectra during the hour of the shock passage.

elements differ from that of oxygen as well. However, it is unclear whether a systematic trend from element to element can be found. While the enhancements of heavy ions (relative to average large SEP event abundances) increase with increasing $Z$ at ULEIS energies for most of the events, this pattern does not continue into the higher SIS energies. In fact, 3 of the 5 events show large depletions of ions heavier than $\mathrm{Ar}$ in the SIS data.

[18] One could argue that event-integrated spectra over more than 3 decades in energy are not useful tools in examining these complicated events. The time-intensity plots (Figure 1) clearly show very dissimilar behavior at 1 and $50 \mathrm{MeV} /$ nucleon. In at least 3 of the events, the lowerenergy intensities are dominated by the peak coincident with a shock passage, while higher-energy particles show a prompt rise peaking before the shock and decaying fairly rapidly. If one assumes that the intensity increase at the shock is a sign of local shock acceleration [Lee, 1983] and that higher energy ions are accelerated much closer to the Sun [Kahler, 1994; Mewaldt et al., 2003], then the inten- 
sities represent an evolution (with decreasing energy) of acceleration from close to the Sun, through the interplanetary medium, to nearly $1 \mathrm{AU}$. It is likely that properties of the shock, such as normal angle and strength or speed, vary as it moves from the Sun to $1 \mathrm{AU}$ as does the magnetic connection between the observer and the shock and the available seed population for acceleration. As both the characteristics of the shock, connection to it, and the composition of the seed population contribute to the spectral shape and composition of the resulting accelerated ions, it is likely that event-integrated spectra over a large energy range will have energy-dependent composition and complicated spectral shapes.

[19] In an effort to determine whether the strong intensity increases near the shock passages contribute to the large differences in the composition seen at high and low energies, Figure 8 shows hourly $\mathrm{Fe} / \mathrm{O}$ ratios at 0.77 and $12 \mathrm{MeV} /$ nucleon for 11 hours around each shock passage. It is clear that even for the events $(2,3$, and 4) with substantial shock effects, the compositional change across the shock is relatively minor (less than a factor of 2 and not systematic from event to event). Since the abundances of the locally accelerated population mirrors that of the abundances observed earlier in the event (at the same energy), the differences between the 0.77 and $12 \mathrm{MeV} /$ nucleon event-integrated composition (Figure 6) are not the result of the fact that some of the events are dominated at low energies by particles accelerated at $1 \mathrm{AU}$ and alternative explanations must be found.

[20] As the material at high energies is presumably accelerated out of a lower-energy population, it is instructive to examine the ratio of the SIS to ULEIS abundances. This is done in Figure 9, where the event-integrated abundances at 12 to $60 \mathrm{MeV} /$ nucleon from the right plots of Figure 6 are normalized by those at 0.64 to $0.91 \mathrm{MeV} /$ nucleon (left plots of Figure 6) and plotted versus $\mathrm{Z}$ and $Q / M$. One would expect processes affecting acceleration and transport of the high-energy particles to be related to $Q / M$ [Ng et al., 2003, and references therein]; however, only limited information is available on SEP charge states at SIS energies [Mewaldt et al., 2005], and no information is available at ULEIS energies, for these events. Instead the average measured charge states obtained in many large SEP events between 0.18 and $0.44 \mathrm{MeV} /$ nucleon [Klecker et al., 2000; Möbius et al., 2000], as given in Table 4, are used. On the other hand, $Z$ is known and generally a monotonic function of $Q / M$, so that is shown in Figure 9 as well. Although event-to-event variation is still apparent, there is a clear trend common to all events (this trend remains if one uses ULEIS abundances at $0.3 \mathrm{MeV} /$ nucleon instead). The heavier ions, or those with smaller $Q / M$, are less efficiently accelerated to higher energies. The magnitude of this effect in $\mathrm{Fe} / \mathrm{O}$ varies by approximately a factor of 3 over the 5 events studied here, but, on average, reflects a $\sim 10 \%$ relative efficiency in accelerating $\mathrm{Fe}$ as compared to $\mathrm{O}$ from $\sim 0.77$ to $\sim 12 \mathrm{MeV} /$ nucleon.

[21] Desai et al. [2004] have determined that higherrigidity ions (e.g., $\mathrm{Fe}$ ) near $1 \mathrm{MeV} /$ nucleon are less efficiently accelerated by interplanetary shocks than lower-rigidity ions (e.g., C). However, this effect is accounted for in the current analysis by normalizing the SIS abundances by those of ULEIS. Thus the depletion in $\mathrm{Fe} / \mathrm{O}$ at $12-60 \mathrm{MeV} /$ nucleon relative to the abundance at $0.64-0.91 \mathrm{MeV} /$ nucleon is in addition to the reduction relative to the source that Desai et al. [2004] found.

[22] The lower abundance of $\mathrm{Fe}$ (relative to $\mathrm{O}$ ) at the higher energies is also seen in Figure 5 and primarily results from the smaller energy of the spectral break (i.e., point at which the spectra begin to soften; $E_{\text {break }}$ ) for iron as compared to that of oxygen (Figure 4 ). The value of $E_{\text {break }}$ is related to the maximum energy to which a shock can accelerate ions, which is, in turn, governed by the diffusion coefficients. Ions with lower $Q / M$ values and higher rigidity (e.g., $\mathrm{Fe}$ ) have larger diffusion coefficients and are less well confined to the shock region and not accelerated to as high energies. Additionally, as the shock moves toward $1 \mathrm{AU}$, it weakens and the maximum energy to which it can accelerate ions decreases [ $\mathrm{Li}$ et al., 2005]. The value of $E_{\text {break }}$ for $\mathrm{Fe}$ is also apparent in the $\mathrm{Fe} / \mathrm{O}$ versus energy plots of Figure 5 , where the decrease in the $\mathrm{Fe} / \mathrm{O}$ with increasing energy typically begins at $E_{\text {break }}(\mathrm{Fe})$. Here it is clear that events 1 , 4 , and 5 (26 October and 2 and 4 November) had lower values of $E_{\text {break }}(\mathrm{Fe})$ than events 2 and 3 (28 and 29 October), suggesting that, for these events, the shock was weaker, there was less turbulence near the shock, or the spacecraft was magnetically connected to a weaker part of the shock (i.e., the flank rather than the nose). This is consistent with the fact that the shock transit times (relative to the CME observed launch times) were nearly twice as long as those of the other 2 events even though the CME speeds (obtained at several solar radii above the Sun) were comparable.

[23] The increase in the $\mathrm{Fe} / \mathrm{O}$ ratios with increasing energy above $10 \mathrm{MeV} /$ nucleon in the events of 2 and 4 November is unexpected, although such behavior has been observed before [Cohen et al., 2003; Tylka et al., 2001]. Suggested explanations for these observations have invoked direct access to flare material [Cane et al., 2003] or reacceleration of flare suprathermals by quasi-perpendicular shocks [Tylka et al., 2005]. However, both these ideas involve $\mathrm{Fe} / \mathrm{O}$ ratios enhanced over the average large SEP event value of 0.134 . Such enhancements are not seen in either the event-integrated abundances or the hourly intensities at energies above $10 \mathrm{MeV} /$ nucleon for these events. Thus it appears that if there is any flare-related contribution to these events it is dominated by shock-accelerated coronal/solar wind material.

[24] Below $\sim 0.3 \mathrm{MeV} /$ nucleon the behavior of Fe and $\mathrm{O}$ is also often different (e.g., events 1, 2, and 5). One aspect appears to be common to nearly all the events; the $\mathrm{Fe} / \mathrm{O}$ ratio approaches the large SEP event average value of 0.134 as the energy decreases. This is similar to trends observed by Mazur et al. [1992] in 10 large SEP events. However, the degree of energy dependence in the $\mathrm{Fe} / \mathrm{O}$ ratio below 0.3 $\mathrm{MeV} /$ nucleon varies from event to event in a manner that is not currently understood. The remainder of this section will focus on the region between 0.3 and $\sim 30 \mathrm{MeV} /$ nucleon which generally contains the breaks in the spectra, features that may be more easily understood.

[25] The cause of the break in the spectra is most likely related to diffusion effects [Ellison and Ramaty, 1985, and references therein] such as escape from the shock region, 

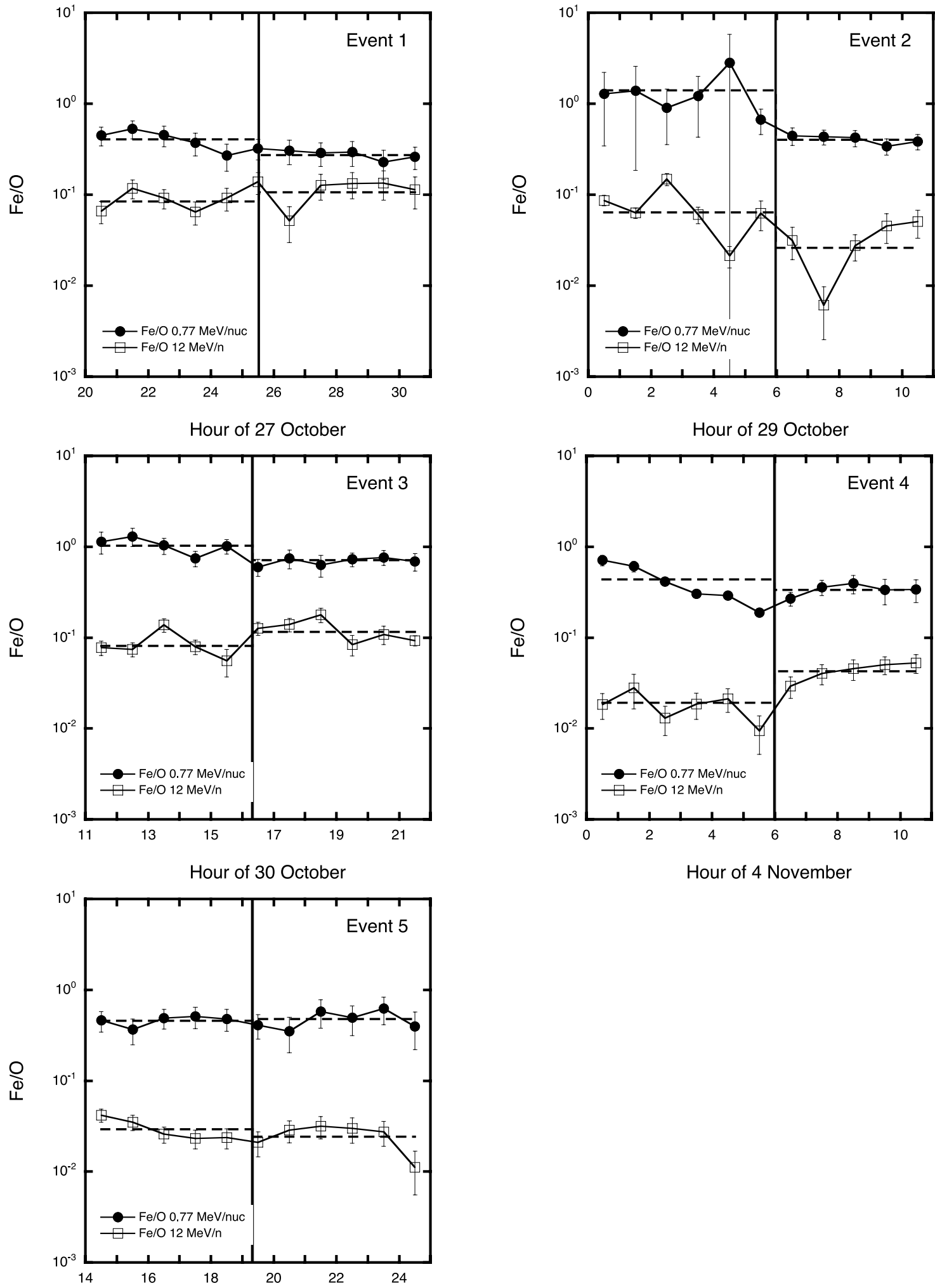

Hour of 4 November

Hour of 6 November

Figure 8. Fe/O ratios from ULEIS at $0.77 \mathrm{MeV} /$ nucleon and from SIS at $12 \mathrm{MeV} /$ nucleon versus time centered around the shock passages during the five SEP events. Dashed lines indicate averages of values before and after shock. 


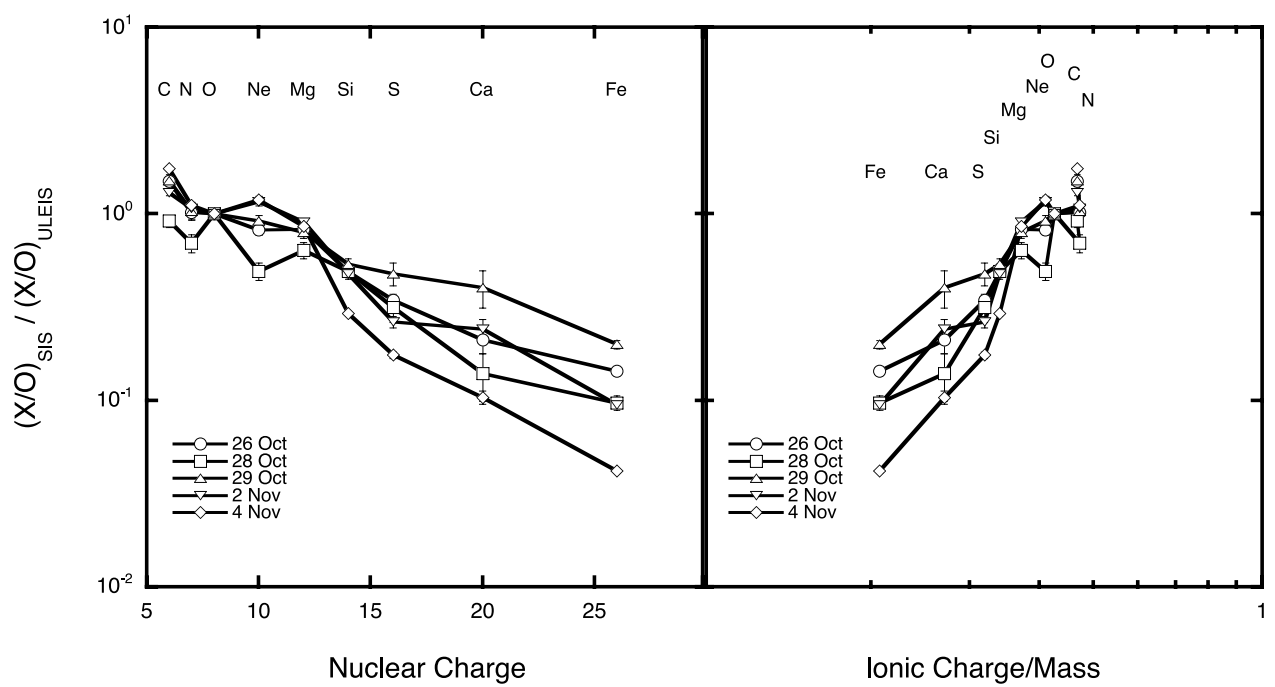

Figure 9. SIS abundances (normalized to oxygen) divided by ULEIS abundances (normalized to oxygen) as a function of (left) nuclear charge and (right) $Q / M$ for the five events.

where the escape distance $l_{\text {esc }}$ can be expressed in terms of the ion diffusion coefficient, $\kappa$, and the shock velocity, $\dot{R}$ [Zank et al., 2000, equation 35],

$$
l_{\text {esc }}=a \frac{\kappa}{\dot{R}} \text {. }
$$

Thus one would expect the relative positions of the breaks for different elements to scale according to their diffusion coefficients, $\kappa$,

$$
\kappa=1 / 3 \mathrm{v} \lambda,
$$

where $v$ is ion velocity and $\lambda$ is the ion mean free path. Assuming $\lambda$ is a power law in rigidity, or $(\mathrm{Mv} / Q)^{\alpha}$ for nonrelativistic particles, one obtains

$$
\kappa \sim(M / Q)^{\alpha}(E)^{(\alpha+1) / 2}
$$

where $E$ is energy per nucleon. The spectral breaks for different species should occur at the same value of the diffusion coefficient and so a scaling in energy between one element (1) and another (2) can be found:

$$
E_{1} / E_{2}=\left[(Q / M)_{1} /(Q / M)_{2}\right]^{2 \alpha /(\alpha+1)} .
$$

Using this relationship, the spectra of $\mathrm{Ne}, \mathrm{Mg}, \mathrm{Si}, \mathrm{S}, \mathrm{Ca}$, and Fe were scaled in energy to that of $\mathrm{O}$ and elemental ratios (relative to $\mathrm{O}$ ) versus scaled energy were calculated for different $\alpha$ values. A single value for $\alpha$ was then selected for each event such that these ratios, between 0.3 and $\sim 30 \mathrm{MeV} /$ nucleon, were relatively flat. Although the energy scaling is specifically appropriate to the position of the spectral breaks, and not the entire spectra, aligning the break points via this scaling has the greatest influence in flattening the elemental ratios. Additionally, one might expect the spectra below the break energy to be determined by the shock characteristics, not diffusion, and therefore be independent of $Q / M$ and so not strongly affected by the scaling given above. A similar application of this scaling to anomalous cosmic ray spectra was done by Cummings et al.
[1984] with success. The oxygen spectra, scaled according to the chosen $\alpha$ values (lines), are compared to the eventintegrated spectra (points) for each event in Figure 10. Considering a single parameter $(\alpha)$ was varied for each event (values of $Q$ were taken from Table 4), the general agreement between the spectra and the scaled oxygen curves in the region of interest is remarkable. The $\alpha$ values are given in Table 5 for reference.

[26] Droege [1994] related the value of $\alpha$ to the turbulence spectrum, assumed to be a power law in wave number, or $k^{-q}: \alpha=2-q$. The values of $\alpha$ obtained here, ranging from 0.8 to 2.7 , suggest the wave spectrum varied from $k^{-1.2}$ to $k^{+0.7}$. That such spectra are flatter than the dispersive region of the interplanetary turbulence spectrum (e.g., a Kolmogorov spectrum of $k^{-5 / 3}$ ) is consistent with a source of turbulence existing near the shock region when these heavy ions were accelerated. When proton-generated waves were incorporated into an SEP transport model, $\mathrm{Ng}$ et al. [2003] found that the wave spectrum at the shock peaked at wave numbers close to where $2.9 \mathrm{MeV} /$ nucleon $\mathrm{Fe}^{13.9+}$ would resonate [see $\mathrm{Ng}$ et al., 2003, Figure 10]. Their results also gave wave spectra with a flat or increasing portion occurring over approximately an order of magnitude in rigidity (corresponding to 2 orders of magnitude in energy). Thus perhaps the rigidity dependences of the mean free path obtained here are not unreasonable.

[27] Elemental ratios can then be taken at common scaled-energy values and are shown for the 5 SEP events

Table 4. Assumed Charge States

\begin{tabular}{ccc}
\hline Element & $\mathrm{Z}$ & $Q^{\mathrm{a}}$ \\
\hline $\mathrm{C}$ & 6 & 5.6 \\
$\mathrm{~N}$ & 7 & 6.6 \\
$\mathrm{O}$ & 8 & 6.8 \\
$\mathrm{Ne}$ & 10 & 8.2 \\
$\mathrm{Mg}$ & 12 & 8.9 \\
$\mathrm{Si}$ & 14 & 9.5 \\
$\mathrm{~S}$ & 16 & 10.2 \\
$\mathrm{Ca}$ & 20 & 10.8 \\
$\mathrm{Fe}$ & 26 & 11.6 \\
\hline
\end{tabular}

${ }^{a}$ Data are taken from Klecker et al. [2000] and Möbius et al. [2000]. 


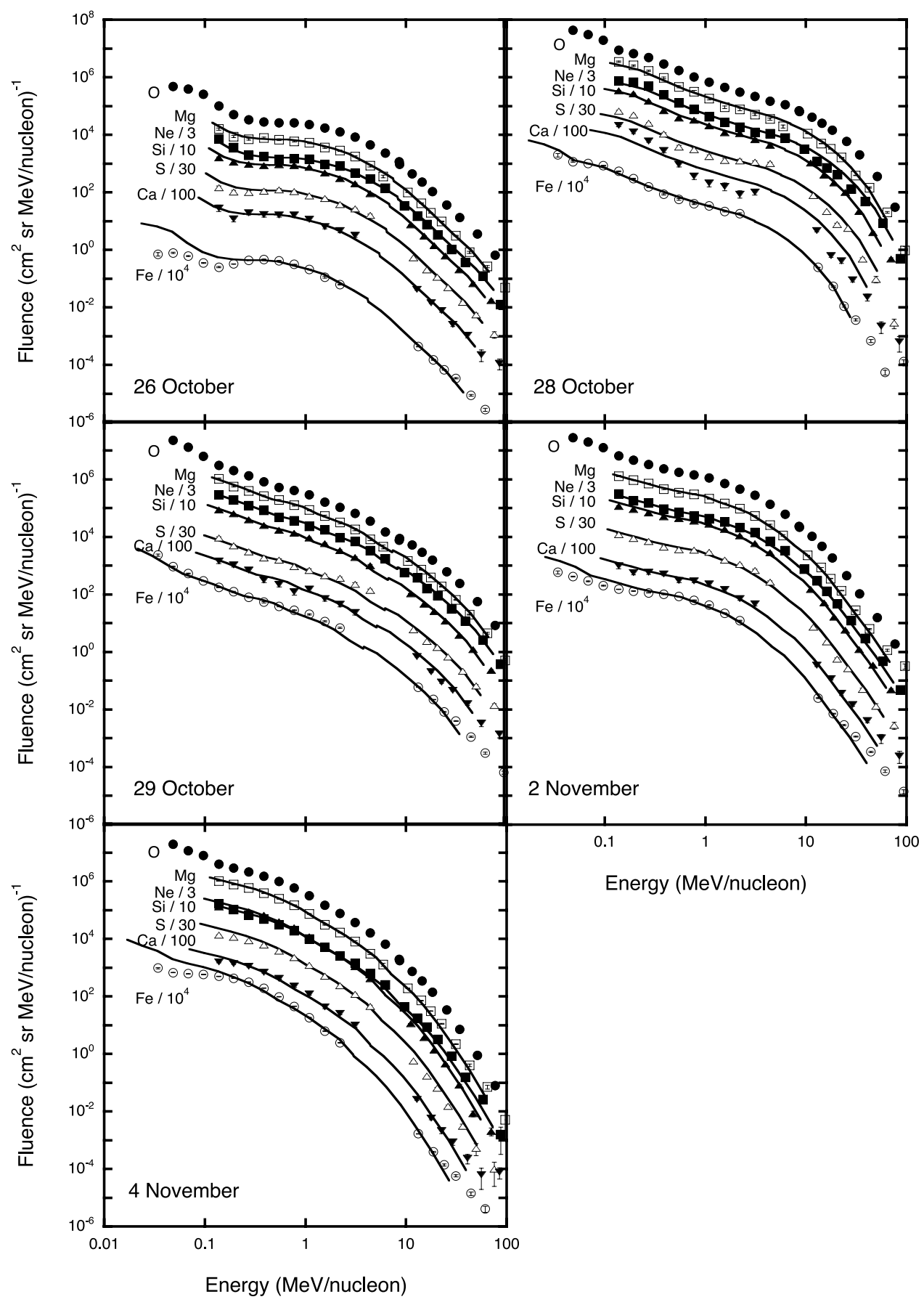

Figure 10. Event-integrated fluences of $\mathrm{O}, \mathrm{Ne}, \mathrm{Mg}, \mathrm{Si}, \mathrm{S}, \mathrm{Ca}$, and Fe plotted versus energy for the five events. All the spectra except $\mathrm{O}$ and $\mathrm{Mg}$ have been scaled to better compare the spectral shapes. The solid lines are the oxygen spectra scaled appropriately in energy (see text).

in Figure 11. The decreasing ratios of $\mathrm{S} / \mathrm{O}$ and $\mathrm{Ca} / \mathrm{O}$ in the 28 October event would only marginally flatten with an increase in $\alpha$, while the $\mathrm{Fe} / \mathrm{O}$ and $\mathrm{Mg} / \mathrm{O}$ ratios would then increase with energy. This different behavior can be seen in the spectra of $\mathrm{S}$ and $\mathrm{Ca}$ in Figure 10 as well, in which both exhibit a flattening between 1 and $10 \mathrm{MeV} /$ nucleon that is not as evident in the other elements.

[28] The high-energy increases in $\mathrm{Fe} / \mathrm{O}$, mentioned previously, seen in the two November events are present in Figure 10 and similar increases are seen in $\mathrm{Ca} / \mathrm{O}$ as well. It is possible that the charge states assumed for the scaling are
Table 5. Fitted Energy Scaling Parameters

\begin{tabular}{cc}
\hline Event & $\alpha$ \\
\hline 1 & 1.0 \\
2 & 2.4 \\
3 & 1.3 \\
4 & 0.8 \\
5 & 2.7 \\
\hline
\end{tabular}




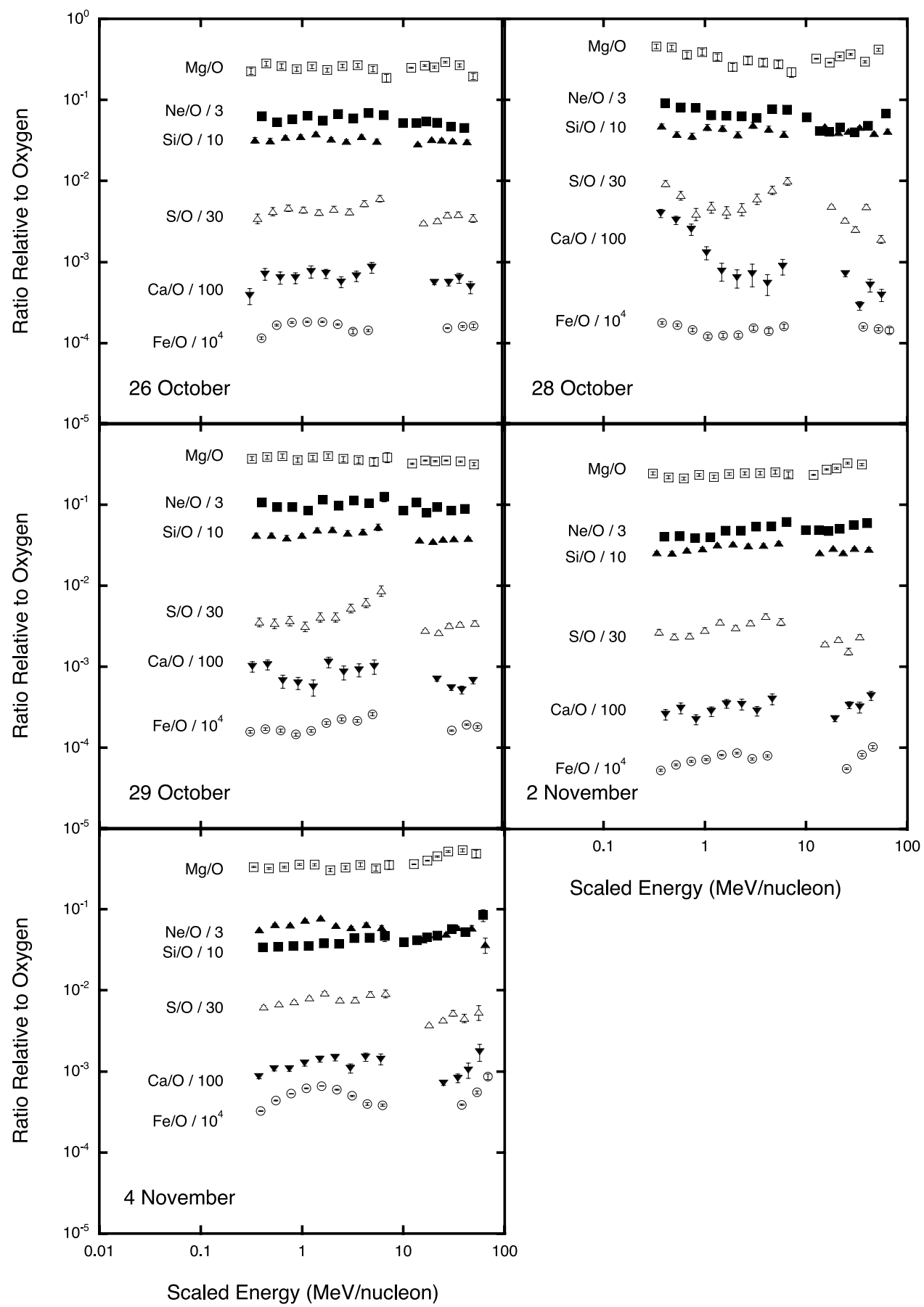

Figure 11. Ratios relative to oxygen, calculated from the spectra in Figure 10, plotted versus scaled energy for the 5 events.

not applicable at these higher energies as there is strong evidence that the charge states in large SEP events are energy-dependent, most noticeably for Fe [Oetliker et al., 1997; Möbius et al., 1999; Mazur et al., 1999]. An increase in the average charge state with energy could result from a series of spectra for individual charge states with break points moving to higher energies for higher charges, which would be combined to produce the overall spectrum. Such a composite spectrum might exhibit a hardening at the higher energies. A similar idea was proposed by Tylka et al. [2001] for the 14 July 2000 SEP event where the authors assumed a distribution of charge states for $\mathrm{Fe}$ that included a 5\% contribution of highly ionized particles (e.g., $\mathrm{Fe}^{17+}$ ). They then constructed an expected Fe spectrum based on the summation of the individual spectra from each charge state and found better agreement with the measured spectrum than when assuming a single charge state of $\sim 10+$.

\section{Conclusions}

[29] The extremely active period of 25 October to 11 November 2003 resulted in 5 readily identified SEP events 
above $10 \mathrm{MeV} /$ nucleon. The observations of the ULEIS and SIS sensors have been combined to obtain elemental spectra and abundances over more than 3 decades in energy. These data indicate that although the second event, starting on 28 October, did not quite have the highest hourly peak oxygen intensity measured by SIS at $10 \mathrm{MeV} /$ nucleon, the eventintegrated fluence surpassed that of all SEP events observed by ACE since launch in August of 1997. However, this event still falls short of the very large event of 19 October 1989 (and presumably that of 4 August 1972 although oxygen intensities were not obtained for this event).

[30] Although 4 of the 5 events studied here are related to flares which originated in the same active region as it moved across the solar disk, the spectra differ substantially from one SEP event to the next. In addition, the Fe and $\mathrm{O}$ fluence spectra plotted versus energy per nucleon for a given event do not have the same shape, resulting in energydependent $\mathrm{Fe} / \mathrm{O}$ ratios for all 5 events. By examining the temporal evolution of the $\mathrm{Fe} / \mathrm{O}$ ratios at two different energies, the effect of local shock acceleration on the event-integrated spectra was evaluated. Little change in the abundance across the shocks was found, indicating the large compositional differences seen in the event-integrated ratios between $0.64-0.91$ and $12-60 \mathrm{MeV} /$ nucleon are related to something else intrinsic to the acceleration and transport processes. When normalized to the low-energy abundances, the 12-60 MeV/nucleon abundances were roughly organized by $Q / M$ for all 5 events.

[31] Focusing on the relative positioning of the spectral breaks, a scaling in energy per nucleon between elements was deduced by assuming the break energy corresponded to a particular value of the diffusion coefficient. When compared according to this scaling, the spectra of $\mathrm{O}, \mathrm{Ne}, \mathrm{Mg}, \mathrm{Si}$, $\mathrm{S}, \mathrm{Ca}$, and $\mathrm{Fe}$ were found to be remarkably similar from 0.3 to $\sim 30 \mathrm{MeV} /$ nucleon. The only free parameter used to align the spectra was $\alpha$ which governs the rigidity dependence of the ions' mean free paths and was assumed to be the same for all elements but allowed to vary from event to event. Values of alpha between 0.8 and 2.7 were obtained for the 5 SEP events and resulted in abundance ratios that were approximately independent of scaled energy. Such values of $\alpha$ imply significantly enhanced wave turbulence in the region of the shock; however, as the inferred value of $\alpha$ is dependent on the assumed charge state of the ions, accurately determining the mean free path dependence on rigidity for these ions requires measurements of the ionic charge state of heavy ions.

[32] The data also suggest the charge state of $\mathrm{Ca}$ and $\mathrm{Fe}$ may increase with increasing energy for the 2 and 4 November events, a characteristic which has certainly been observed in other events. Thus knowledge of the charge states of heavy ions, particularly as a function of energy, is key to understanding the abundance variations seen in large SEP events. The charge state information may also provide additional clues regarding the seed populations being accelerated close to the Sun and through the interplanetary medium.

[33] Acknowledgments. This work was supported by NASA at the California Institute of Technology (under grant NAG5-6912), the Jet Propulsion Laboratory, NASA Goddard Space Flight Center, and in part under Caltech grant 44A1055749 at the University of Maryland.
[34] Arthur Richmond thanks Eberhard S. Moebius and another reviewer for their assistance in evaluating this paper.

\section{References}

Cane, H. V., T. T. von Rosenvinge, C. M. S. Cohen, and R. A. Mewaldt (2003), Two components in major solar particle events, Geophys. Res. Lett., $30(12), 8017$, doi:10.1029/2002GL016580.

Cohen, C. M. S., R. A. Mewaldt, A. C. Cummings, R. A. Leske, E. C. Stone, T. T. von Rosenvinge, and M. E. Wiedenbeck (2003), Variability of spectra in large solar energetic particle events, Adv. Space Res., 32, $2649-2654$

Cook, W. R. (1981), Elemental composition of solar energetic particles, Ph.D. thesis, Calif. Inst. of Technol., Pasadena.

Cummings, A. C., and E. C. Stone (1998), Anomalous cosmic rays and solar modulation, Space Sci. Rev., 83, 51-62.

Cummings, A. C., E. C. Stone, and W. R. Webber (1984), Evidence that the anomalous cosmic-ray component is singly ionized, Astrophys. J., 287, L99-L103.

Desai, M. I., et al. (2004), Spectral properties of heavy ions associated with the passage of interplanetary shocks at $1 \mathrm{AU}$, Astrophys. J., 611, 11561174.

Droege, W. (1994), Transport of solar energetic particles, Astrophys. J. Suppl. Ser., 90, 567-576.

Ellison, D. C., and R. Ramaty (1985), Shock acceleration of electrons and ions in solar flares, Astrophys. J., 298, 400-408.

Garrard, T. L., and E. C. Stone (1991), Heavy ions in the October 1989 solar flares observed on the Galileo spacecraft, paper presented at 22nd International Cosmic Ray Conference, Inst. for Adv. Stud., Dublin.

Gopalswamy, N., S. Yashiro, Y. Liu, G. Michalek, A. Vourlidas, M. L. Kaiser, and R. A. Howard (2005), Coronal mass ejections and other extreme characteristics of the 2003 October-November solar eruptions, J. Geophys. Res., doi:10.1029/2004JA010958, in press.

Kahler, S. (1994), Injection profiles of solar energetic particles as functions of coronal mass ejection heights, Astrophys. J., 428, 837-842.

Klecker, B., et al. (2000), Comparison of ionic charge states of energetic particles with solar wind charge states in CME related events, AIP Conf. Proc., 528, 135-138.

Lee, M. A. (1983), Coupled hydromagnetic wave excitation and ion acceleration at interplanetary traveling shocks, J. Geophys. Res., 88, 61096119.

Li, G., G. P. Zank, and W. K. M. Rice (2005), Acceleration and transport of heavy ions at coronal mass ejection-driven shocks, J. Geophys. Res., 110, A06104, doi:10.1029/2004JA010600.

Mason, G. M., et al. (1998), The Ultra-Low-Energy Isotope Spectrometer (ULEIS) for the ACE spacecraft, Space Sci. Rev, 86, 409-448.

Mason, G. M., et al. (1999), Particle acceleration and sources in the November 1997 solar energetic particle events, Geophys. Res. Lett., 26, $141-144$

Mason, G. M., J. R. Dwyer, and J. E. Mazur (2000), New properties of ${ }^{3} \mathrm{He}$-rich solar flares deduced from low-energy particle spectra, Astrophys. J., 545, L157-L160.

Mazur, J. E., G. M. Mason, B. Klecker, and R. E. McGuire (1992), The energy spectra of solar flare hydrogen, helium, oxygen, and iron: Evidence for stochastic acceleration, Astrophys. J., 401, 398-410.

Mazur, J. E., G. M. Mason, M. D. Looper, R. A. Leske, and R. A. Mewaldt (1999), Charge states of solar energetic particles using the geomagnetic cutoff technique: SAMPEX measurements in the 6 November 1997 solar particle event, Geophys. Res. Lett., 26, 173-176.

Mewaldt, R. A., et al. (2003), Heavy ion and electron release times in solar particle events, in Proceedings of the 4th IGPP Conference, edited by T. Kajita et al., pp. 3313-3316, Universal Acad. Press, Tokyo.

Mewaldt, R. A., et al. (2005), Solar energetic particle spectra breaks, in Physics of Collisionless Shocks: Proceedings of the 4th IGPP Conference, AIP Conf. Proc., edited by G. Li et al., Inst. Geophys. and Planet. Phys., Riverside, Calif., in press.

Möbius, E., et al. (1999), Energy dependence of the ionic charge state distribution during the November 1997 solar energetic particle event, Geophys. Res. Lett., 26, 145-148.

Möbius, E., et al. (2000), Survey of ionic charge states of solar energetic particle events during the first year of ACE, AIP Conf. Proc., 528, 131134.

Ng, C. K., D. V. Reames, and A. J. Tylka (2003), Modeling shockaccelerated solar energetic particles coupled to interplanetary Alfven waves, Astrophys. J., 591, 461-485.

Oetliker, M., B. Klecker, D. Hovestadt, G. M. Mason, J. E. Mazur, R. A. Leske, R. A. Mewaldt, J. B. Blake, and M. D. Looper (1997), The ionic charge of solar energetic particles with energies of $0.3-70 \mathrm{MeV}$ per nucleon, Astrophys. J., 477, 495-501, doi:10.1086/303679. 
Reames, D. V. (1999), Particle acceleration at the Sun and in the heliosphere, Space Sci. Rev., 90, 413-491.

Reames, D. V., and C. K. Ng (1998), Streaming-limited intensities of solar energetic particles, Astrophys. J., 504, 1002-1005.

Stone, E. C., et al. (1998a), The solar isotope spectrometer for the Advanced Composition Explorer, Space Sci. Rev., 86, 357-408.

Stone, E. C., A. M. Frandsen, R. A. Mewaldt, E. R. Christian, D. Margolies, J. F. Ormes, and F. Snow (1998b), The Advanced Composition Explorer, Space Sci. Rev., 86, 1-22.

Tylka, A. J. (2001), New insights on solar energetic particles from Wind and ACE, J. Geophys. Res., 106, 25,333-25,352.

Tylka, A. J., C. M. S. Cohen, W. F. Dietrich, C. G. Maclennan, R. E McGuire, C. K. Ng, and D. V. Reames (2001), Evidence for remnant flare suprathermals in the source population of solar energetic particles in the 2000 Bastille Day event, Astrophys. J., 558, L59-L63.

Tylka, A. J., C. M. S. Cohen, W. F. Dietrich, M. A. Lee, C. G. Maclennan, R. A. Mewaldt, C. K. Ng, and D. V. Reames (2005), Shock geometry, seed populations, and the origin of variable elemental composition at high energies in large gradual solar particle events, Astrophys. J., 625, $474-495$.
Williams, D. L. (1998), Measurements of the isotopic composition of solar energetic particles with the MAST instrument aboard the SAMPEX spacecraft, Ph.D. thesis, Calif. Inst. of Technol., Pasadena.

Zank, G. P., W. K. M. Rice, and C. C. Wu (2000), Particle acceleration and coronal mass ejection driven shocks: A theoretical model, J. Geophys. Res., 105, 25,079-25,096.

C. M. S. Cohen, A. C. Cummings, R. A. Leske, R. A. Mewaldt, and E. C. Stone, Space Radiation Laboratory, California Institute of Technology, MC 220-47, Pasadena, CA 91125, USA. (cohen@srl.caltech.edu; ace@srl. caltech.edu; ral@srl.caltech.edu; rmewaldt@srl.caltech.edu; ecs@srl. caltech.edu)

M. I. Desai and G. M. Mason, Department of Physics, University of Maryland, College Park, MD 20742, USA. (desai@uleis.umd.edu; glenn.mason@umail.um.edu)

T. T. von Rosenvinge, NASA Goddard Space Flight Center, Code 661, Greenbelt, MD 20771, USA. (tycho@milkyway.gsfc.nasa.gov)

M. E. Wiedenbeck, Jet Propulsion Laboratory, 4800 Oak Grove Dr., M/S 169-327, Pasadena, CA 91109, USA. (mark.e.wiedenbeck@jpl.nasa.gov) 